\title{
Power Reduction of Chip Multi-Processors using Shared Resource Control Cooperating with DVFS
}

\author{
Ryo Watanabe, Masaaki Kondo, Hiroshi Nakamura, Takashi Nanya \\ Research Center for Advanced Science and Technology, \\ The University of Tokyo \\ 4-6-1 Komaba, Meguro-ku, Tokyo, Japan \\ \{watanabe,kondo,nakamura,nanya\}@ hal.rcast.u-tokyo.ac.jp
}

\begin{abstract}
This paper presents a novel power reduction method for chip multi-processors (CMPs) under real-time constraints. While the power consumption of processing units (PUs) on CMPs can be reduced without violating real-time constraints by dynamic voltage and frequency scaling (DVFS), the clock frequency of each PU cannot be determined independently because of the performance impact caused by the conflict for the shared resources. To minimize power consumption in this situation, we first derive an analytical model which provides the optimal priority and clock frequency setting, and then propose a method of controlling the priority of shared resource accesses in cooperation with DVFS. From the analytical model, in dual-core CMPs, we reveal that the total power consumption is minimized when the clock frequency of two PUs becomes the same. An experiment with a synthetic benchmark supports the validity of the analytical model and the evaluation results with real applications show that the proposed method reduces the power consumption by up to $15 \%$ and $6.7 \%$ on average compared with a conventional DVFS technique.
\end{abstract}

\section{Introduction}

To reduce power consumption, and thus reduce energy consumption, dynamic voltage and frequency scaling (DVFS) is widely used, especially in real-time systems. By lowering the clock frequency and supply voltage, the power consumption of a processor is reduced while satisfying the real-time constraint.

Recently, the single chip multiprocessor (CMP) has become an attractive architecture. With multiple processors, we can achieve high throughput without increasing clock frequency; thereby, CMP offers higher power-performance efficiency compared to that of single processor systems.

In CMPs, multiple PUs are often used to improve the performance of multi-program workloads. When PUs execute several independent applications which have different real-time constraints, the suitable clock frequency and voltage for each application should be selected to reduce power consumption, if each PU can choose its own clock frequency and supply voltage independently of other PUs. However, the clock frequency of PUs cannot be determined independently, even if independent programs are executed. This is because of the existence of the shared resources conflict.

In order to utilize limited resources efficiently, PUs on CMPs usually share some hardware components such as the L2 cache, memory bus and main memory. If two or more PUs try to use such shared resources simultaneously, all but one of the PUs have to wait until the ongoing access finishes. The performance penalty (waiting time) due to the conflict heavily depends on the access patterns of running programs. As the performance penalty increases, PUs must use a higher clock frequency and supply voltage to compensate for the performance loss and to keep satisfying the performance constraint. Thus, the power consumption of PUs also heavily depends on the occurrence of the conflict in shared resources. This implies that the power consumption of PUs is altered if we control the effect of the performance penalty caused by the conflict.

In this paper, we propose a method to minimize the power consumption of CMPs by priority control in accessing shared resources. The performance penalty caused by the resource conflict can be altered by the priority control. Suppose that we have two PUs (denoted as $\mathrm{PU}_{A}$ and $\mathrm{PU}_{B}$ ) and the shared resource accesses from $\mathrm{PU}_{A}$ have a higher priority than those from $\mathrm{PU}_{B}$. Then, the waiting time due to the resource conflict of $\mathrm{PU}_{A}$ decreases, and consequently, $\mathrm{PU}_{A}$ can use a lower clock frequency, which leads to lower power consumption of $\mathrm{PU}_{A}$. On the other hand, the waiting time of $\mathrm{PU}_{B}$ increases, and therefore the clock frequency of $\mathrm{PU}_{B}$ must be increased to satisfy the performance constraint. Clearly, the power consumption of $\mathrm{PU}_{B}$ 
increases. There should be an optimal priority setting which minimizes the total power consumption, and the proposed method tries to find the optimal priority setting as well as the clock frequency for each PU.

This paper makes the following contributions.

- First, we derive the condition in which the total power consumption becomes minimal by constructing a model which analyzes the performance and power impact caused by the shared resource conflict and priority control.

- Second, we propose a method which cooperatively optimizes the access priority and the clock frequency of each PU based on a proposed model. This method can be easily implemented in conventional CMPs. In this paper, we assumes that the priority control for shared resource accesses is realized by a priority queue.

The rest of this paper is organized as follows. The next section briefly presents the strategy for power reduction using the access priority control. Section 3 clarifies the condition of power minimization with an analytical model. In Section 4, we propose a cooperative priority and clock frequency control method and in Section 5 we show some experimental results. We describe related work in Section 6 and conclude in Section 7.

\section{Strategy for Power Reduction}

\subsection{Problem Settings}

We investigate a power minimization problem in a typical dual-core CMP which concurrently executes two programs with real-time constraints. We suppose that the target CMP consists of two identical PUs, $\mathrm{PU}_{0}$ and $\mathrm{PU}_{1}$. Each PU has dedicated L1 and L2 caches and can operate with an individual clock frequency and supply voltage denoted as $f_{i}$ and $V_{i}$, respectively. Here, the subscript $i$ indicates the index of the PUs. All the PUs access the main memory through a shared memory bus.

$\mathrm{PU}_{i}$ is supposed to execute an application task $\mathrm{T}_{\mathrm{i}}$. As mentioned in the previous section, application tasks on different PUs are independent and $T_{i}$ has its own real-time constraint (or latency constraint) which is denoted as $L_{i}$. This means that the execution of one iteration of $T_{i}$ must be completed within time $L_{i}$. Note that parallel tasks are not considered in this paper.

\subsection{Strategy}

The key strategies for power minimization are as follows: 1) deriving the optimal priority setting which minimizes the total power consumption by constructing a performance and power model, 2) controlling the priority of the

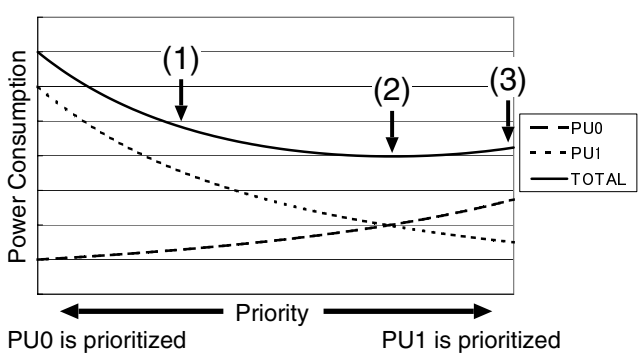

Figure 1. Effect of the priority control.

shared resource access in order to alter the power consumption of each PU. We explain the effect of the priority control with the example shown in Figure 1. The figure shows the relationship between the priority setting and power consumption of the PUs. This graph is derived by simulating the formulated model described in Section 3.

In Figure 1, the latency constraint of $\mathrm{T}_{1}$ is supposed to be tighter than that of $\mathrm{PU}_{0}$. Therefore, $\mathrm{PU}_{1}$ has to operate with a higher clock frequency than $\mathrm{PU}_{0}$ to satisfy the constraint. Consequently, the power consumption of $\mathrm{PU}_{1}$ is larger than that of $\mathrm{PU}_{0}((1)$ in Figure 1).

As stated in Section 1, the clock frequency and supply voltage required to meet the constraint can be altered by priority control of the shared resource. When the access from $\mathrm{PU}_{1}$ is prioritized, $\mathrm{PU}_{1}$ can decrease its clock frequency because of the shorter waiting time. On the other hand, $\mathrm{PU}_{0}$ has to increase its clock frequency to compensate for the increasing performance penalty.

The power consumption of the PUs increases quadratically as the clock frequency increases [6]. Therefore, the reduction of power in $\mathrm{PU}_{1}$ (which uses a higher clock frequency) is larger than the increase of power in $\mathrm{PU}_{0}$ (which uses a lower clock frequency). For this reason, total power consumption of the CMP decreases with the prioritizing of accesses from $\mathrm{PU}_{1}$. This trend continues until the power reduction of $\mathrm{PU}_{1}$ becomes equal to the power increase of $\mathrm{PU}_{0}$ ((2) in Figure 1). As will be discussed in Section 3, this is the point at which the clock frequency of $\mathrm{PU}_{0}$ is equal to that of $\mathrm{PU}_{1}$.

When the access priority of $\mathrm{PU}_{1}$ is increased beyond this point, the trend of the total power consumption begins to increase ((3) in Figure 1). This is because the increase in power for $\mathrm{PU}_{0}$ becomes larger than the reduction of power for $\mathrm{PU}_{1}$ as the clock frequency of $\mathrm{PU}_{0}$ becomes larger than that of $\mathrm{PU}_{1}$. Therefore, the total power consumption is a convex function of the access priority; in addition, the total power consumption is minimized when the clock frequency of both PUs becomes the same.

In this paper, we assume the memory bus is the only shared resource of the target CMP. However, the strategy presented in this section is applicable to CMPs which have other shared resources. The essence of the strategy is that 
the power consumption of the PUs is altered by means of the priority control so that the total power is minimized.

\section{Conflict Modeling}

This section presents a model for analyzing the performance and power impact of the access priority control on a shared memory bus. From the model, we can obtain the exact priority setting that minimizes the total power consumption of a CMP.

\subsection{Parameters and Variables}

As stated in Section 2, we use the variables $L_{i}, f_{i}$, and $V_{i}$ for the latency constraint, clock frequency, and supply voltage for each PU, respectively. In addition to these variables, we introduce a parameter which indicates the performance of the shared memory bus.

- $l_{B}$ : Bus access latency (time for transferring data from main memory to L2 cache)

We also introduce some parameters for representing the task properties, as follows.

- $I_{i}$ : Number of instructions for $T_{\mathrm{i}}$

- $m_{i}$ : Number of $\mathrm{L} 2$ misses for $\mathrm{T}_{\mathrm{i}}$

- $s_{i}$ : Length of stall time in $\mathrm{PU}_{i}$ due to $\mathrm{L} 2$ cache misses when executing $\mathrm{T}_{\mathrm{i}}$

These parameters are assumed to be provided by techniques such as the static analysis of the execution profiles of programs, on-line estimation, and so on. Note that the analytical model discussed later does not depend on how these parameters are extracted.

Using the above assumptions, the effective working time of each PU is given as follows.

$$
t_{i}=L_{i}-s_{i}
$$

The effective working time means the length of time that a PU really performs useful computations. Thus, $\mathrm{PU}_{i}$ has to complete execution of $I_{i}$ instructions within time $t_{i}$. The processing speed of $\mathrm{PU}_{i}$ required to keep the deadline depends on $I_{i}$ and $t_{i}$. Since it has already been shown that theoretically the unique power-optimal clock frequency and voltage level can be determined [8], the power-optimal clock frequency is given as the following formula using a task dependent constant $c$.

$$
f_{i}=c \frac{I_{i}}{t_{i}}
$$

The relationship between the supply voltage and clock frequency is approximated by equation (3).

$$
V_{i}=a f_{i}+b
$$

Table 1. Relationship between clock frequency and supply voltage.

\begin{tabular}{|c|c|c|c|}
\hline Processor & $\begin{array}{c}\text { Max./Min. } \\
\text { Clock Freq. } \\
{[\mathrm{GHz}]}\end{array}$ & $\begin{array}{c}\text { Regression } \\
\text { Formula } \\
\mathrm{V}[\mathrm{V}]=\mathrm{a}[\mathrm{GHz}]+\mathrm{b}\end{array}$ & $\begin{array}{c}\text { Coefficient of } \\
\text { Determination } \\
\mathrm{R}^{2}\end{array}$ \\
\hline \hline Pentium M [10] & $1.6 / 0.6$ & $\mathrm{~V}=0.558 \mathrm{f}+0.609$ & 0.9920 \\
\hline Pentium M [2] & $2.1 / 0.6$ & $\mathrm{~V}=0.237 \mathrm{f}+0.848$ & 0.9997 \\
\hline Turion 64 [1] & $2.0 / 0.8$ & $\mathrm{~V}=0.250 \mathrm{f}+0.700$ & 1.0000 \\
\hline
\end{tabular}

This linear relationship is observed in many commercial processors. Table 1 shows some examples of the relationship. Using regression analysis, the relationship between the clock frequency and supply voltage is almost a linear function, as shown in the third column in Table 1. Because the coefficient of determination (the fourth column in the table) is very high, this approximation seems to be fully reasonable.

Then, using system dependent constant $k$, the energy consumption per instruction for $\mathrm{PU}_{\mathrm{i}}$, denoted as $e_{i}$, is derived as follows [6].

$$
e_{i}=k V_{i}^{2}
$$

Finally, the average power consumption of $\mathrm{PU}_{i}$ is presented below.

$$
P_{i}=\frac{I_{i}}{L_{i}} e_{i}=\frac{k I_{i} V_{i}^{2}}{L_{i}}
$$

\subsection{Impact of Access Conflicts}

In this subsection, we formulate the power and performance impact of memory bus conflicts when $T_{0}$ and $T_{1}$ are executed simultaneously. During the execution of $\mathrm{T}_{\mathrm{i}}$, the length of time that $\mathrm{PU}_{i}$ occupies the bus is given by $m_{i} l_{B}$. If L2 misses have uniform distribution, the probability that $\mathrm{PU}_{1}$ occupies the bus when $\mathrm{PU}_{0}$ tries to access it is as follows.

$$
p_{0}=\frac{m_{1} l_{B}}{L_{1}}
$$

This also indicates the probability of occurrence of $\mathrm{PU}_{0}$ 's stall due to the bus conflict. Once the conflict occurs, the data transfer of $\mathrm{PU}_{0}$ on the bus must wait for completion of the ongoing data transfer requested by $\mathrm{PU}_{1}$. The expectation of the time length that $\mathrm{PU}_{0}$ waits for the bus to be available is shown in equation (7).

$$
E_{w}=\frac{1}{2} l_{B}
$$

From (6) and (7), the expectation of additional stall time per $\mathrm{L} 2$ cache miss for $\mathrm{PU}_{0}$ is given as $P_{0} E_{w}$. Thus, the effective working time of $\mathrm{PU}_{0}$ decreases to the value shown in equation (8).

$$
t_{0}^{\prime}=t_{0}-m_{0} p_{0} E_{w}=t_{0}-m_{0} \frac{m_{1} l_{B}^{2}}{2 L_{1}}
$$


The power-optimal clock frequency and supply voltage can be obtained in the same way as stated in the previous section, and is described as follows.

$$
f_{0}^{\prime}=c \frac{I_{0}}{t_{0}^{\prime}}, V_{0}^{\prime}=a f_{0}^{\prime}+b, e_{0}^{\prime}=k V_{0}^{\prime 2}
$$

The same procedure can also be applied for $\mathrm{PU}_{1}$ to derive $t_{1}^{\prime}, f_{1}^{\prime}, V_{1}^{\prime}$ and $e_{1}^{\prime}$. Thus, the average power consumption of $\mathrm{PU}_{i}$, including the impact of the shared bus conflict, is given as follows.

$$
P_{i}^{\prime}=\frac{I_{i}}{L_{i}} e_{i}^{\prime}=\frac{k I_{i} V_{i}^{\prime 2}}{L_{i}}
$$

\subsection{Power Reduction by Priority Control}

In this subsection, we formulate the effect of the access priority control.

\subsubsection{Distributing the Performance Penalty}

From equation (8), the total extra stall time caused by the conflict per unit of time for all PUs can be derived as follows.

$$
l_{\text {total }}=\frac{1}{L_{0}} \frac{m_{0} m_{1} l_{B}^{2}}{2 L_{1}}+\frac{1}{L_{1}} \frac{m_{0} m_{1} l_{B}^{2}}{2 L_{0}}=\frac{m_{0} m_{1} l_{B}^{2}}{L_{0} L_{1}}
$$

Here, we suppose the system has an access priority controller. When an access conflict happens, the controller chooses one request among several requests from $\mathrm{PU}_{0}$ and $\mathrm{PU}_{1}$ based on the priority setting. Note that we cannot reduce the total amount of the performance penalty $l_{\text {total }}$ even with the priority control. The priority control can only alter the allotment of the performance penalties among PUs.

Suppose that the priority controller allocates the penalty so that the ratio of the penalty for each $\mathrm{PU}$ is $\mathrm{PU}_{0}: \mathrm{PU}_{1}=$ $r:(1-r)(0 \leq r \leq 1)$. In this case, the effective working time of each PU is expressed as the following formulas.

$$
\begin{array}{r}
t_{0}^{\prime}=t_{0}-L_{0} r l_{\text {total }} \\
t_{1}^{\prime}=t_{1}-L_{1}(1-r) l_{\text {total }}
\end{array}
$$

From these formulas, it is shown that the total power consumption of the CMP, expressed as $P_{\text {total }}\left(=P_{0}^{\prime}+P_{1}^{\prime}\right)$, is a function of the variable $r$.

\subsubsection{Power Optimization}

Here, we derive a condition in which the total power consumption of the CMP is minimized.

When the supply voltage $V$ is represented as a linear function of the clock frequency, as in equation (3), $V^{2}$ is a convex polynomial function of $f$ and is also a convex function of $r$ (from equations (9), (12) and (13)). Hence, $P_{\text {total }}$ is also a convex function of $r$ and has a global minimum, as shown in Figure 1.
Using the derivative constants $c_{2}, c_{1}, c_{0}$ and equation (9), we express $e_{i}^{\prime}$ as follows.

$$
e_{i}^{\prime}=c_{2}\left(\frac{I_{i}}{t_{i}^{\prime}}\right)^{2}+c_{1}\left(\frac{I_{i}}{t_{i}^{\prime}}\right)+c_{0}
$$

The convex function becomes the minimum when the derivative is equal to zero. Here, the following formula for the derivative of $P_{\text {total }}$ holds.

$$
\begin{gathered}
\quad \frac{d}{d r} P_{\text {total }}=\frac{I_{0}}{L_{0}} \frac{d}{d r} e_{0}^{\prime}+\frac{I_{1}}{L_{1}} \frac{d}{d r} e_{1}^{\prime} \\
=l_{\text {total }}\left\{2 c_{2}\left(\frac{I_{0}}{t_{0}^{\prime}}\right)^{3}+c_{1}\left(\frac{I_{0}}{t_{0}^{\prime}}\right)^{2}\right. \\
\left.-2 c_{2}\left(\frac{I_{1}}{t_{1}^{\prime}}\right)^{3}-c_{1}\left(\frac{I_{1}}{t_{1}^{\prime}}\right)^{2}\right\}
\end{gathered}
$$

This immediately leads to the result of the condition for minimizing the total power consumption, which is represented as follows.

$$
\frac{I_{0}}{t_{0}^{\prime}}=\frac{I_{1}}{t_{1}^{\prime}} \quad \Rightarrow \quad f_{0}^{\prime}=f_{1}^{\prime}
$$

This formula indicates that the total power consumption of the CMP is minimized by controlling the access priority so that the each clock frequency of each PU becomes equal. Therefore, the value of $r$ which minimizes the total power consumption of the CMP is derived as follows.

$$
r_{\text {min }}=\frac{I_{1} t_{0}-I_{0} t_{1}+I_{0} L_{1} l_{\text {total }}}{\left(I_{0} L_{1}+I_{1} L_{0}\right) l_{\text {total }}}
$$

In some cases, $r_{\min }$, given by equation (17), can be more than 1 or less than 0 . Even in this case, the power is minimized by making the clock frequency of the PUs close to each other. Therefore, we use 1 in the case of $r_{\min }>1$, and 0 in the case of $r_{\min }<0$.

\subsection{Case for CMPs with More PUs}

Although we focus on a dual-core CMP in this paper, the model presented in this section can be easily extended to CMPs with more PUs. We briefly describe how to extend the model.

Suppose $N$ PUs $\left(\mathrm{PU}_{0}, \mathrm{PU}_{1}, \ldots, \mathrm{PU}_{\mathrm{N}-1}\right)$ share the memory bus. The probability of $p_{i}$ (the probability that access from $\mathrm{PU}_{i}$ is delayed due to the bus conflict) is given as the probability that any of the other PUs occupies the bus at that moment. This is shown in equation (18). From this equation, the power and performance impact caused by the access conflict can be derived in the same way as in the case of two PUs.

$$
p_{i}=\sum_{j \neq i} \frac{m_{j} l_{B}}{L_{j}}
$$




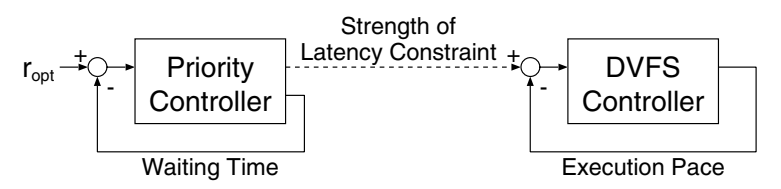

Figure 2. Block diagram of the cooperative priority and DVFS control method.

Here, $l_{\text {total }}$ can also be expressed using equation (18,) and it is a constant even with access priority control. Therefore, power minimization is a problem for allocating the performance penalty among $N$ PUs.

Suppose that the ratio of the penalty is $\mathrm{PU}_{0}: \mathrm{PU}_{1}: \ldots$ : $\mathrm{PU}_{\mathrm{N}-2}: \mathrm{PU}_{\mathrm{N}-1}=r_{0}: r_{1}: \ldots: r_{N-2}:\left(1-r_{0}-r_{1}-\right.$ $\left.\ldots-r_{N-2}\right)\left(\forall i 0 \leq r_{i} \leq 1\right)$. In this situation, the partial derivative of $P_{\text {total }}$ with respect to $r_{i}$ is derived similarly to equation (15), because the power consumption of the PUs is constant except for that of $\mathrm{PU}_{i}$ and $\mathrm{PU}_{\mathrm{N}-1}$. This leads to the conclusion that the total power consumption of $\mathrm{PU}_{i}$ and $\mathrm{PU}_{\mathrm{N}-1}$ becomes a minimum when $f_{i}=f_{N-1}$.

By applying the above process for all $i(0 \leq i \leq N-$ 2 ), the relationship $f_{0}=f_{1}=\ldots=f_{N-1}$ is derived as the condition for power minimization. Thus, even in CMPs with more than two PUs, the settings for priority control that enables power minimization is derived in the same way as in the case of two PUs.

\section{Control Method}

\subsection{Overview}

Figure 2 illustrates an overview of the proposed cooperative priority and clock frequency control method. The two cooperating feedback controllers in this method minimize the power consumption of the CMP based on the derived condition presented in Section 3.

The priority controller located on the left side of Figure 2 adjusts the priority for conflicting accesses so that the ratio of the penalty caused by the conflict for the PUs comes close to the power-optimal ratio $\left(r_{\min }: 1-r_{\min }\right)$, which is the input of the priority controller. The value of $r_{\min }$ is calculated by equation (17). The DVFS controller located on the right side of the figure adjusts the clock frequency and supply voltage of each PU so that the performance constraint of all the PUs is satisfied. The tightness of the latency constraint for each PU, which is the input to the controller, is derived as a result of the priority controller.

In the following sections, we describe the details of the priority control and the DVFS control for a dual-core CMP.

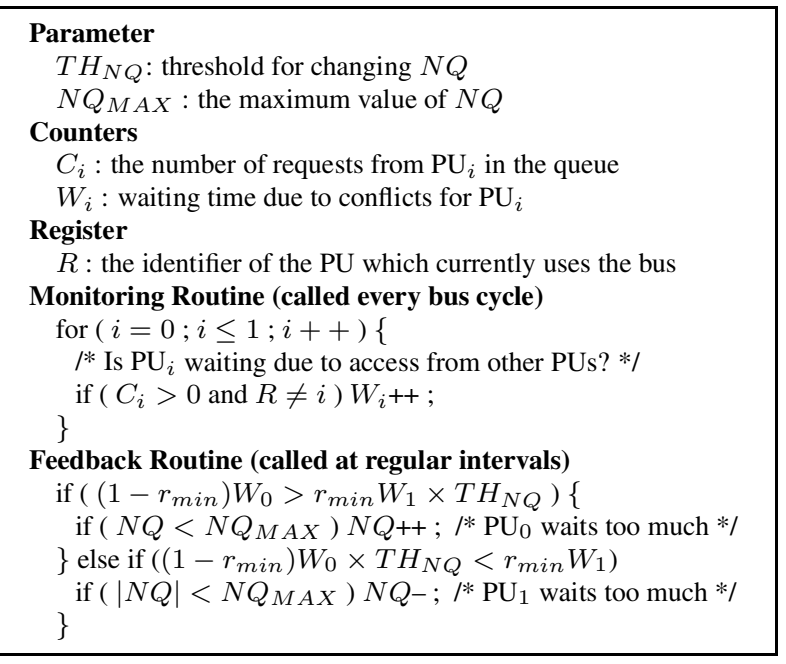

Figure 3. Algorithm of the priority control.

\subsection{Priority Control}

The priority control is based on access management with a priority queue. The queues are popular hardware used for managing accesses to shared resources. All of the access requests to the memory (and to the memory bus) due to $\mathrm{L} 2$ cache misses in any of the PUs are queued and processed one by one. To use a queue as a priority queue, we introduce an integer parameter $N Q$. If $N Q>0$, a request from $\mathrm{PU}_{0}$ is granted before previously generated $N Q$ requests from $\mathrm{PU}_{1}$. On the other hand, if $N Q<0$, a request from $\mathrm{PU}_{1}$ is granted before previously generated $|N Q|$ requests from $\mathrm{PU}_{0}$. Otherwise, the requests in the queue are selected based on first-come-first-served (FCFS) manner.

Although we try to allocate the performance penalty to each PU by the priority control with the priority queue, the optimal ratio of the penalty is difficult to achieve because it is not directly handled by the priority control method mentioned above. Therefore, we also introduce a feedback control mechanism which updates the priority setting of the queue according to the observed performance penalty ratio as well as the target penalty ratio. The algorithm of the feedback priority control is shown in Figure 3. In this algorithm, the amount of waiting time due to conflict is monitored for each PU. Based on the actual performance penalty that is corrected for each PU, the new value of priority $N Q$ is decided so that the actual penalty ratio becomes close to the target penalty ratio.

\subsection{DVFS Control}

As mentioned in Section 3.3.2, once the task parameters are given, the clock frequency which minimizes the total power consumption is derived from the model together with $r_{\min }$. However, the assumptions used to derive the 


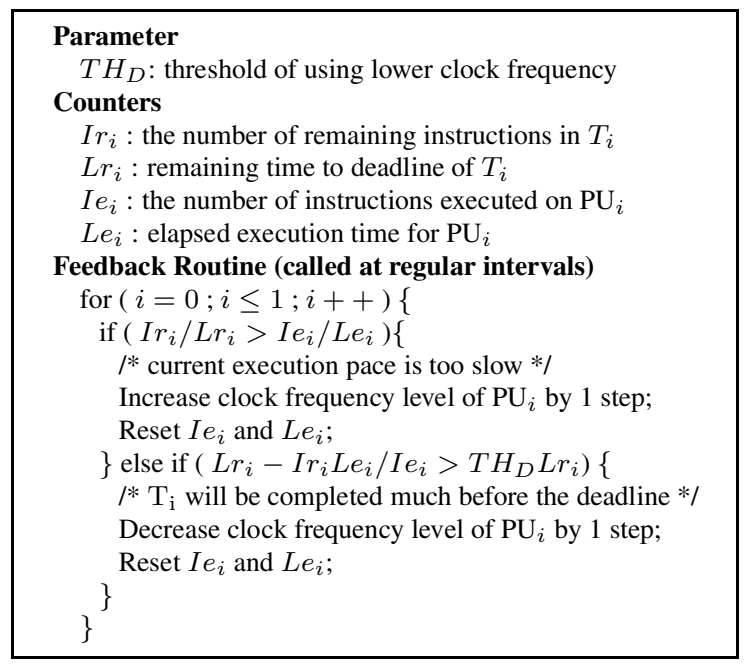

Figure 4. Algorithm of the DVFS control.

model are slightly inapplicable to typical CMP systems for the following reasons: 1 ) the number of available clock frequency levels is limited, 2) the target $r_{\min }$ is sometimes not achievable because all the access conflicts are not controllable, even with the priority control method. Therefore, we propose the DVFS method, which also includes a feedback control mechanism. The algorithm is shown in Figure 4.

In the algorithm, the current processing speed is checked for each task at regular intervals and the current speed is compared with the target speed required to complete the task just within its deadline. Then the clock frequency for the next interval is decided.

\section{Evaluation}

We evaluated the proposed method to validate the proposed model and to show the effectiveness of the method. We used SimpleScalar Tool Set [3] as the base simulation environment. We extended SimpleScalar so that the assumed CMP architecture was evaluated. For estimating the power consumption, we used the Wattch [4] extension.

The hardware configuration and parameters for the proposed algorithm are shown in Table 2 and Table 3, respectively. The relationship between clock frequency and supply voltage was assumed to follow the linear approximation of that of Pentium M [10], as shown in Table 1.

\subsection{Model Validation}

We evaluated a synthetic application to verify the following two results derived by the model analysis: 1) the total power consumption is reduced by making the clock frequency of the PUs closer, 2) the power is minimized by controlling the access priority when the ratio of the performance penalty becomes $\mathrm{PU}_{0}: \mathrm{PU}_{1}=r_{\text {min }}:\left(1-r_{\text {min }}\right)$. The
Table 2. Hardware configuration.

\begin{tabular}{|l||c|}
\hline PU clock frequency & $200 \sim 1600 \mathrm{MHz}$ \\
\hline PU supply voltage & $0.721 \sim 1.502 \mathrm{~V}$ \\
\hline Fetch, Issue, Commit width & 4 \\
\hline Branch prediction & $\begin{array}{c}\text { Combined bimodal (4K-entry) } \\
\text { gshare (1K-entry), selector(1K-entry) }\end{array}$ \\
\hline BTB & 1,024 sets, 2-way \\
\hline Mis-prediction penalty & 5 cycles \\
\hline Load / Store Queue size & 16 \\
\hline Reorder buffer size & 32 \\
\hline Functional units & (INT) 2-ALU, 1-mult/div \\
& (FP) 2-FPU, 2-mult/div \\
& (MEM) 1-port \\
\hline L1 I-cache & $32 \mathrm{~KB}, 32 \mathrm{~B}$ line, 2-way \\
& 1 -cycle latency \\
\hline L1 D-cache & $32 \mathrm{~KB}, 32 \mathrm{~B}$ line, 2-way \\
& 2 -cycle latency \\
\hline L2 unified cache & $256 \mathrm{~KB}, 64 \mathrm{~B} \mathrm{line,} \mathrm{4-way}$ \\
& 10 -cycle latency \\
\hline Memory latency & $100 \mathrm{~ns}$ \\
\hline Bus width & $8 \mathrm{~B}$ \\
\hline Bus clock & $200 \mathrm{MHz}$ \\
\hline
\end{tabular}

Table 3. Algorithm parameters.

\begin{tabular}{|l||c|}
\hline Priority feedback interval & $1.87 \mathrm{us}$ \\
\hline DVFS feedback interval & $187 \mathrm{us}$ \\
\hline$N Q_{M A X}$ & 3 \\
\hline$T H_{N Q}$ & 1.01 \\
\hline$T H_{D}$ & 0.05 \\
\hline
\end{tabular}

synthetic application had a constant L2 miss rate throughout the execution, while the interval of consecutive L2 misses follows the Poisson distribution. In this experiment, we assumed DVFS with a very large number of available clock frequency levels. This is for making the condition almost the same as the hardware assumption used in the analytical model. We evaluated several patterns of the combination of L2 miss rates and latency constraints.

The evaluation results are shown in Table 4. For each evaluated case, we present the L2 miss rate, the average clock frequency without priority control (this indicates the strength of the latency constraint), $r_{\min }$ derived from the model, the actual waiting time ratio achieved by the control, the average clock frequency when priority control is applied, and the degree of power reduction achieved by the proposed method.

This result shows that the power consumption of the CMP is reduced by controlling the access priority. The degree of power reduction depends on the strength of the latency constraint and the $\mathrm{L} 2$ miss rate. As for case 1 and case 2 in the table, $r_{\min }$ cannot be achieved even by the priority control. This is due to the limitation of priority controllability of the queue-based method. However, even in such a situation, the proposed method can reduce the power consumption by reducing the difference in the clock frequency of each PU.

Figure 5 shows the changes in the power consumption in 
Table 4. Results with a synthetic program for model validation.

\begin{tabular}{|c|c|c|c|c|c|c|c|c|c|}
\hline \multirow[t]{2}{*}{$\begin{array}{l}\text { Evaluated } \\
\text { Case }\end{array}$} & \multicolumn{2}{|c|}{ L2 miss rate $[\%]$} & \multicolumn{2}{|c|}{$\begin{array}{l}\text { Avg. Freq. of PUs [MHz] } \\
\text { only DVFS is applied }\end{array}$} & \multirow[t]{2}{*}{$\begin{array}{l}r_{\min } \text { derived } \\
\text { from the model }\end{array}$} & \multirow{2}{*}{$\begin{array}{c}\text { ratio of } W_{0} \& W_{1} \\
\text { achieved by } \\
\text { the control }\end{array}$} & \multicolumn{2}{|c|}{$\begin{array}{l}\text { Avg. Freq. of PUs [MHz] } \\
\text { priority control is applied }\end{array}$} & \multirow[t]{2}{*}{$\begin{array}{c}\text { Power } \\
\text { reduction [\%] }\end{array}$} \\
\hline & $\overline{\mathrm{PU}_{0}}$ & $\mathrm{PU}_{1}$ & $\mathrm{PU}_{0}$ & $\mathrm{PU}_{1}$ & & & $\mathrm{PU}_{0}$ & $\mathrm{PU}_{1}$ & \\
\hline 1 & $\overline{c 1.76}$ & $\overline{0.91}$ & $\overline{810}$ & 1322 & 1.000 & 0.734 & 8884 & 1226 & 3.5 \\
\hline 2 & 1.76 & 1.76 & 800 & 1312 & 0.837 & 0.736 & 930 & 1038 & 8.4 \\
\hline 3 & 1.76 & 2.71 & 802 & 1308 & 0.645 & 0.655 & 928 & 980 & 10.4 \\
\hline 4 & 2.71 & 2.71 & 910 & 1258 & 0.594 & 0.598 & 1080 & 1070 & 6.6 \\
\hline 5 & 1.76 & 3.36 & 802 & 1268 & 0.626 & 0.623 & 928 & 934 & 11.1 \\
\hline
\end{tabular}

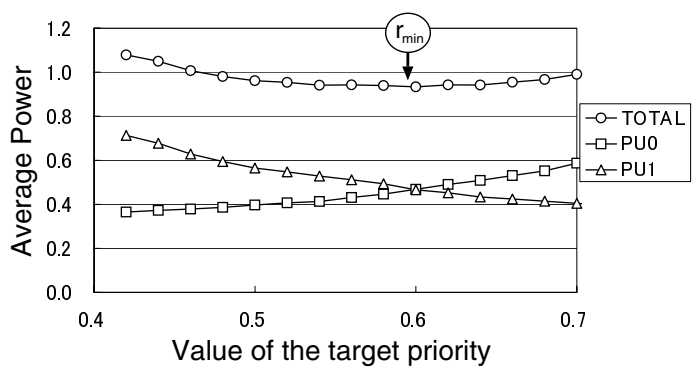

Figure 5. Relationship between power consumption and priority setting.

case 4 in Table 4 when the target value of the priority ratio is varied. The average power shown in the figure is normalized to the case that the proposed method is not used. The figure shows that the power consumption of the CMP is really a convex function of the variable $r$, and the value which minimizes the power is almost the same as $r_{\min }$, given by the proposed analytical model.

\subsection{Evaluation with Real Applications}

We applied the proposed method to real applications. We used the H.264/AVC decoder reference software [7] along with several programs from SPEC CPU2000 benchmark [12]. In this experiment, we assumed DVFS with eight levels of clock frequency and supply voltage. The clock frequency ranged from $200 \mathrm{MHz}$ to $1600 \mathrm{MHz}$ with $200 \mathrm{MHz}$ steps. We fast-forwarded the initialization part of each application and performed the simulation until 1.2 billion instructions of H.264 were completed. The constraint for $\mathrm{PU}_{0}$ (H.264) was assumed to be tighter than that of $\mathrm{PU}_{1}$.

In real applications, the L2 cache miss rate is not uniform throughout the execution. Therefore, we partitioned the target applications into small tasks so that the $\mathrm{L} 2$ cache miss rate could be assumed to be uniform within the tasks. In this case, the combinations of tasks which are simultaneously executed on the PUs change as the execution progresses. Therefore, $r_{\min }$ should be re-calculated whenever the combination changes, as shown in Figure 6).

The evaluation results are shown in Figure 7. This fig-

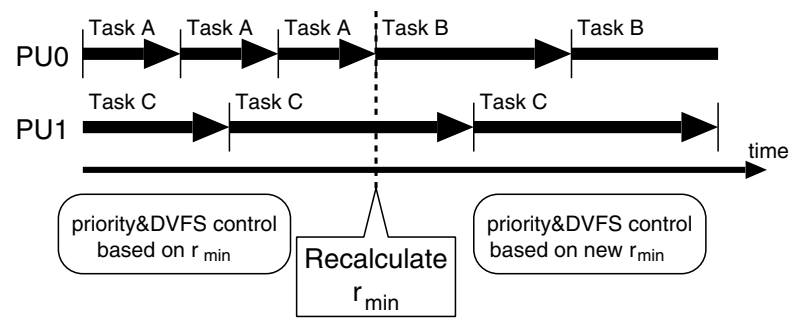

Figure 6. Priority control when combination of executing tasks changes.

ure presents the power consumption of the CMP with the proposed priority and DVFS control methods relative to the CMP, to which only the DVFS algorithm was applied. We evaluated two cases of latency constraints for $\mathrm{PU}_{1}$, which are expressed by the required instruction throughput (instructions per second: IPS) in the figure.

As seen from the figure, the proposed method reduces the power consumption of the CMP by up to $15 \%$ and $6.7 \%$ on average. The effect on the power reduction varies when the latency constraint is varied. For example, in H.264+art, the reduction of power in the case of IPS $=147 \mathrm{M}$, which indicates a tight constraint, is larger than the case of IPS $=132 \mathrm{M}$. This is because more bus conflicts occur when the latency constraint is tight. On the other hand, in H.264+mcf, the case of the looser latency constraint (IPS=89M) can save more power consumption compared with the tighter latency constraint (IPS=93M). Because the gap between the used clock frequency is large for a looser constraint, we have more potential to reduce power.

Figure 8 shows the utilized clock frequency for both PUs when H.264 and mcf (IPS=89M) are executed simultaneously. Figure 8-(1) shows the case when the priority control is not applied, and Figure 8-(2) shows the case when it is applied. Each point in the figure shows the average clock frequency for $200 \mathrm{~ms}$ time intervals. As seen from the figures, we see that the clock frequency of both PUs becomes closer by applying priority control. This is the expected behavior and this indicates that the proposed control method works very well. 


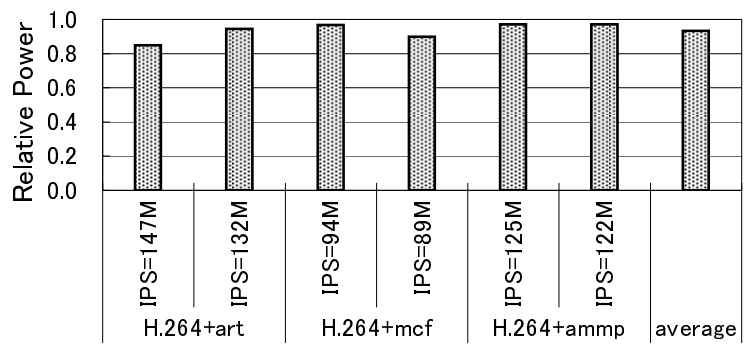

Figure 7. Results with real applications.

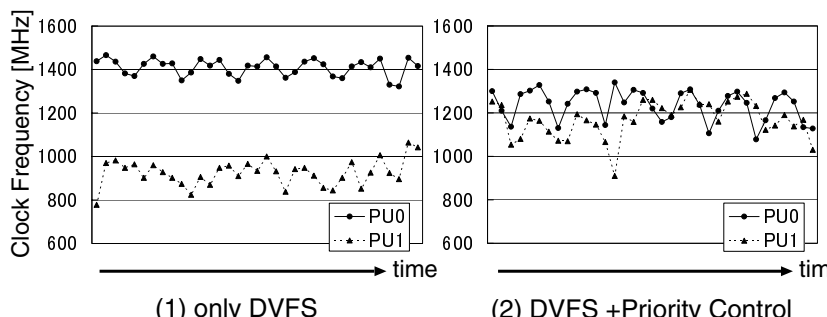

\section{Figure 8. Used clock frequency throughout execution in H.264+mcf (IPS=89M).}

\section{Related Work}

There has been a tremendous effort directed toward the DVFS technique to reduce power consumption. Many of these efforts have attempted to find the optimal choice of clock frequency and voltage level, as in [8], under a real-time constraint. Our work differs from these efforts since we use priority control in cooperation with the DVFS method.

There have been proposed many techniques to alleviate the performance impact caused by the resource conflicts. L2 cache partitioning algorithms have been presented to reduce the overall miss rate on a CMP [13] [9]. A performance model that predicts the impact of cache sharing is presented in [5]. In addition to share cache management, the problems with shared main memory resources is addressed in [11].

Resource conflicts waste power as well as degrade performance in CMPs. The proposed method in this paper provides power reduction by priority control and DVFS for real-time systems when resource conflicts occur. The method should be very useful for future CMP based realtime systems.

\section{Conclusion}

We have proposed a method of access priority control of shared resources in cooperation with the DVFS technique to reduce power consumption for CMPs. We derived an analytical model that provided the optimal priority and clock frequency, and described a feedback-based priority and clock frequency control method. The evaluation results reveal that the analytical model accurately provides the optimal priority, and the proposed cooperative priority and DVFS method can reduce power consumption compared with the conventional power reduction technique that uses only DVFS. Future work includes evaluating the proposed method in a CMP with three or more PUs and investigating the effectiveness of the method with a wide variety of real-time applications.

\section{Acknowledgment}

This work was supported in part by STARC (Semiconductor Technology Academic Research Center) and by Japan Science and Technology Agency as a CREST research program entitled "Innovative Power Control for Ultra Low-Power and High-Performance System LSIs."

\section{References}

[1] AMD Athlon 64 Processor Power and Thermal Data Sheet. http://www.amd.com/us-en/assets/content_type/ white_papers_and_tech_docs/30430.pdf.

[2] Intel Pentium M Processor on 90nm Process with 2-MB L2 Cache Datasheet. http://developer.intel.com/design/mo bile/datashts/302189.htm.

[3] T. M. Austin, E. Larson, and D. Ernst. Simplescalar: An infrastructure for computer system modeling. IEEE Computer, 35(2):59-67, Feb. 2002.

[4] D. Brooks, V. Tiwari, and M. Martonosi. Wattch: a framework for architectural-level power analysis and optimizations. In 27th ISCA, pages 83-94, June 2000.

[5] D. Chandra, F. Guo, S. Kim, and Y. Solihin. Predicting interthread cache contention on a chip multi-processor architecture. In 11th HPCA, pages 340-351, 2005.

[6] A. P. Chandrakasan, S. Sheng, and R. W. Brodersen. Lowpower CMOS digital design. IEEE J. of Solid-State Circuits, 27(4):473-484, Apr. 1992.

[7] H.264/AVC Software Coordination. H.264/AVC reference software. http://iphome.hhi.de/suehring/tml.

[8] T. Ishihara and H. Yasuura. Voltage scheduling problem for dynamically variable voltage processors. In ISLPED 1998, pages 197-202, Aug. 1998.

[9] S. Kim, D. Chandra, and Y. Solihin. Fair cache sharing and partitioning in a chip multiprocessor architecture. In 13th PACT, pages 111-122, Oct. 2004

[10] K. Krewell. Pentium M hits the street. Microprocessor Report, Mar. 2003.

[11] K. J. Nesbit, N. Aggarwal, J. Laudon, and J. E. Smith. Fair queuing memory systems. In 39th MICRO, pages 208-222, Dec. 2006.

[12] Standard Performance Evaluation Corporation (SPEC). SPEC CPU2000. http://www.specbench.org.

[13] G. E. Suh, S. Devadas, and L. Rudolph. A new memory monitoring scheme for memory-aware scheduling and partitioning. In 8th HPCA, pages 117-128, Feb. 2002. 